Copyright by the American Physical Society. Lee, T. K.; Kivelson, S., "Dielectric function of polyacetylene," Phys. Rev. B 29, 6687 DOI: http://dx.doi.org/10.1103/PhysRevB.29.6687

\title{
Dielectric function of polyacetylene
}

\author{
T. K. Lee \\ Department of Physics, Virginia Polytechnic Institute and State University, Blacksburg, Virginia 24061 \\ S. Kivelson \\ Department of Physics, State University of New York at Stony Brook, Stony Brook, New York 11794
}

(Received 16 January 1984)

\begin{abstract}
The optical dielectric function for polyacetylene has been calculated in the random-phase approximation. Local-field corrections are included. The optical-absorption coefficient obtained is in good agreement with experimental data. The contributions of interchain coupling and exchange interaction are discussed.
\end{abstract}

\section{INTRODUCTION}

The physical properties of quasi-one-dimensional organic semiconductors, of which polyacetylene $\left(\mathrm{CH}_{x}\right)$ is one of the most interesting examples, have been the subject of intensive study. By using a simple one-electron model, Su, Schrieffer, and Heeger ${ }^{1}$ (SSH) demonstrated the possible existence of solitons in $\left(\mathrm{CH}_{x}\right)$. Strong experimental evidence of the existence of solitons has been deduced from optical-absorption ${ }^{2}$ and photoinduced absorption data. ${ }^{3}$ Several theoretical calculations ${ }^{4-6}$ of the optical-absorption data have been carried out based on the one-electron model. However, none of this data has taken into account the long-range Coulomb interactions between electrons.

In this paper we calculate the dielectric response func$\operatorname{tion}^{7,8} \epsilon\left(\overrightarrow{\mathrm{q}}+\overrightarrow{\mathrm{G}}, \overrightarrow{\mathrm{q}}+\overrightarrow{\mathrm{G}}^{\prime}, \omega\right)\left(\overrightarrow{\mathrm{G}}\right.$ and $\overrightarrow{\mathrm{G}}^{\prime}$ are reciprocal-lattice vectors) of a simple model of a quasi-one-dimensional semiconductor. The model consists of a threedimensional array of weakly coupled chains. We assume that the noninteracting electronic structure on each chain can be described by the SSH Hamiltonian corresponding to a perfectly dimerized $(\mathrm{CH})_{x}$ chain. We further assume that the atomic orbitals are highly localized; overlap between the wave functions on neighboring sites is neglected or treated as small in all of our considerations. Although this model is obviously of particular relevance for $(\mathrm{CH})_{x}$, it should be a fairly good representation of a wide class of conducting polymers. Our principle conclusions are as follows.

(1) We obtain a formal expression for the microscopic dielectric function $\epsilon\left(\overrightarrow{\mathrm{q}}+\overrightarrow{\mathrm{G}}, \overrightarrow{\mathrm{q}}+\overrightarrow{\mathrm{G}}^{\prime}, \omega\right)$, both with and without taking into account exchange interactions. Note that $\epsilon$ is a matrix reflecting the fact that the crystal only has discrete translational invariance.

(2) We obtain an explicit expression for the macroscopic dielectric function $\epsilon(\overrightarrow{\mathrm{q}}, \omega)$ in the long-wavelength limit $q a \ll 1$, where $a$ is a lattice constant along the chain. This function is evaluated at three levels of approximation. First, we calculate $\epsilon_{\mathrm{RPA}}^{(0)}(\overrightarrow{\mathrm{q}}, \omega)$ using the randomphase approximation (RPA) but without taking local-field effects into account. Second, by taking local-field effects into account we obtain a qualitatively different dielectric function $\epsilon_{\mathrm{RPA}}(\overrightarrow{\mathrm{q}}, \omega)$. Third, we estimate the correction to $\epsilon_{\mathrm{RPA}}$ due to exchange, and argue that it is probably quite small.

(3) We have examined the contribution to $\epsilon_{\mathrm{RPA}}^{(0)}$ of the weak interchain coupling. We find that the function $\epsilon_{\mathrm{RPA}}^{(0)}$ can be expressed as a Taylor series in the anisotropy parameter $X_{\perp}=4 t_{\perp} / E_{g}$ [see Eqs. (2c) and (19)], where $E_{g}$ is the in-chain (one-dimensional) semiconducting gap and $4 t_{\perp}$ is the transverse bandwidth. The first term in the series which depends on $X_{\perp}$ is the quadratic term. Thus, even in systems which are only moderately anisotropic, the dielectric function can be calculated to a fair degree of accuracy ignoring interchain coupling. This also means that the dielectric function is largely insensitive to the details of the three-dimensional structure of the material other than the density of chains.

(4) We show that the correction to the imaginary part of $\epsilon_{\mathrm{RPA}}^{(0)}(\vec{q}, \omega)$ due to the overlap of the atomic wave functions from neighboring sites is negligible for $\omega$ near the band gap $E_{g}$. The correction only becomes important when $\omega$ is much larger than the band gap.

(5) The contribution to the dielectric function $\epsilon_{\mathrm{RPA}}(\overrightarrow{\mathrm{q}}, \omega)$ due to a local-field effect can be characterized by one dimensionless parameter $V_{c}$ [defined in Eq. (30)]. $V_{c}$ is a ratio of some kind of average Coulomb energy to the in-chain bandwidth $4 t_{0}$.

(6) The optical-absorption coefficient is calculated by using $\epsilon_{\mathrm{RPA}}^{(0)}$ and $\epsilon_{\mathrm{RPA}}$, and both are compared with experimental data (see Fig. 2). There is a good agreement for the latter case, $\epsilon_{\mathrm{RPA}}$. The result is quite insensitive to the precise value of the parameter $V_{c} / 4 t_{0}$.

(7) Our results for the static dielectric function can be summarized simply as

$\epsilon_{\mathrm{RPA}}^{(0)}(\overrightarrow{\mathrm{k}}, 0)=\epsilon_{0}+\epsilon_{1}\left(\frac{k_{\|}}{k}\right)^{2}\left[F(y)+O\left(X^{2}\right)+\left(\frac{k_{\|} a}{y}\right)^{2}\right]$,

where

$$
\epsilon_{1}=\frac{4 e^{2} a^{2}}{3 \Omega_{0} t_{0}}\left[\frac{1}{y}\right)^{2}
$$


$k_{\|}$is the component of $\overrightarrow{\mathrm{k}}$ in the in-chain direction, $y \equiv E_{g} / 4 t_{0}$ is assumed to be much less than $1, F(y)$ is a function of order $1[F(0)=1]$ defined in Eq. (20), and $\Omega_{0}$ is the volume of the unit cell. Local-field and exchange interactions only affect this result slightly [see Eq. (32)].

The plan of this paper is as follows. In Sec. II the oneelectron model of $(\mathrm{CH})_{x}$ is defined and the one-electron band structure is discussed. In Sec. III, a formal expression for the dielectric function is derived. In Sec. IV the formal expressions for the dielectric function are evaluated at the various levels of approximation, and this section also contains our principle results. All three of these sections and the appendix are rather heavily mathematical, although we have tried to catalog the results in Sec. IV as clearly as possible. In Sec. $\mathrm{V}$ we discuss the implications of our results in the specific case of solid polyacetylene. Finally, Sec. VI contains a few general conclusions.

\section{ONE-ELECTRON MODEL OF (CH) $x$}

A schematic representation of a piece of trans- $(\mathrm{CH})_{x}$ crystal is shown in Fig. 1. We notice that there are two inequivalent sites (carbons) on each chain which we refer to as even- and odd-numbered sites. In the schematic crystal structure shown in Fig. 1, all of the chains are equivalent and hence there are two sites per unit cell. The actual crystal structure of $(\mathrm{CH})_{x}$ contains two inequivalent chains per unit cell ${ }^{9}$ and possibly also considerable disorder. For our purposes, these are unimportant complications. Thus, we will assume that all chains are equivalent and thus each site is labeled by a lattice vector $\overrightarrow{\mathbf{R}}$ and an index, $v$, which is 0 for even sites and 1 for odd sites. Following SSH $^{1}$ we consider a model which includes one $\pi$ orbital per site which forms a half-filled $\pi$ band. This band is split in two by the bond alternation. We describe this system by the simplest possible threedimensional generalization of the SSH Hamiltonian,

$$
H=\sum_{l} H_{l}+H_{\perp}
$$

where $H_{l}$ is the single-chain Hamiltonian for chain $l$,

$$
H_{l}=-t_{0} \sum_{n, s}\left[1+(-1)^{n} y\right]\left(C_{l, n, s}^{\dagger} C_{l, n+1, s}+\text { H.c. }\right),
$$

$C_{l, n, s}^{\dagger}$ creates an electron of spin $s$ on site $n$ of chain $l, y$ is a measure of the magnitude of the bond alternation, and $H$ is the interchain coupling which we take to be of the<smiles>C=C/C=C\C=C/C=C\C=C/C=C\C</smiles>

$\begin{array}{llllllll}\text { Site \# } & 1 & 2 & 3 & 4 & 5 & 6 & 7\end{array}$

FIG. 1. Schematic crystal structure of trans- $(\mathrm{CH})_{x}$. simple form

$$
H_{\perp}=-t_{\perp} \sum_{\left\langle l, l^{\prime}\right\rangle}\left(C_{l, n, s}^{\dagger} C_{l^{\prime}, n, s}+\text { H.c. }\right) \text {. }
$$

In terms of the site and chain indices, we can take the Bravais lattice to be the points $\vec{R}$ which specify the location of the even-numbered sites. The eigenstates of this Hamiltonian are of the form

$$
\langle\overrightarrow{\mathrm{r}} \mid \overrightarrow{\mathrm{k}}, v, s\rangle=\frac{1}{\sqrt{N}} \sum_{\overrightarrow{\mathrm{R}}} e^{i \overrightarrow{\mathrm{k}} \cdot \overrightarrow{\mathrm{R}}} \sum_{v=0}^{1} U_{v}^{n}(\overrightarrow{\mathrm{k}}) \phi_{v}(\overrightarrow{\mathrm{r}}-\overrightarrow{\mathrm{R}}-v \overrightarrow{\mathrm{a}}),
$$

where $n=0$ or 1 for the conduction- and valence-band states, respectively, and $\phi_{v}(\overrightarrow{\mathrm{r}}-\overrightarrow{\mathrm{R}}-v \overrightarrow{\mathrm{a}})$ is the Wannier function associated with the site at $\vec{R}+v \vec{a}$, and $\vec{a}$ is the displacement vector between the even and odd sites in the same unit cell. The wave functions $U_{v}^{\mu}(\overrightarrow{\mathrm{k}})$ are easily found to depend only on $k_{\|}$according to the relation

$$
\begin{aligned}
& U_{v}^{0}(\overrightarrow{\mathrm{k}})=\frac{1}{\sqrt{2}}\left[(-1)^{v} \alpha\left(k_{\|}\right)-i \beta\left(k_{\|}\right)\right] e^{i v k_{\|} a}, \\
& U_{v}^{1}(\overrightarrow{\mathrm{k}})=\frac{1}{\sqrt{2}}\left[\alpha\left(k_{\|}\right)-i(-1)^{v} \beta\left(k_{\|}\right)\right] e^{i v k_{\|^{a}}},
\end{aligned}
$$

where

$$
\begin{aligned}
& \left.\alpha(k)=\left\{s_{\|}(k)+\cos (k a)\right] / s_{\|}(k)\right\}^{1 / 2}, \\
& \beta(k)=\operatorname{sgn}(k)\left\{\left[s_{\|}(k)-\cos (k)\right] / s_{\|}(k)\right\}^{1 / 2},
\end{aligned}
$$

and

$$
s_{\|}(k)=\left[1-\left(1-y^{2}\right) \sin ^{2}(k)\right]^{1 / 2} .
$$

From these expressions it is easy to see that the energy eigenvalues are

$$
E_{n}(\overrightarrow{\mathrm{k}})=(-1)^{n} 2 t_{0} s_{\|}\left(k_{\|}\right)+2 t_{\perp} s_{\perp}\left(\overrightarrow{\mathrm{k}}_{\perp}\right),
$$

where $\overrightarrow{\mathrm{k}}_{\perp}$ is the component of $\overrightarrow{\mathrm{k}}$ in the plane perpendicular to the chain direction, and $s_{\perp}$ is the interchain structure factor

$$
s_{\perp}\left(\overrightarrow{\mathrm{k}}_{\perp}\right)=\frac{1}{2} \sum_{\overrightarrow{\mathrm{l}}}^{\prime} e^{i \overrightarrow{\mathrm{k}}_{\perp} \cdot \overrightarrow{\mathrm{R}}_{l}},
$$

where the sum is over the nearest neighbors of a particular chain and $\overrightarrow{\mathbf{R}}_{l}$ is the displacement to the $l$ th chain.

It is clear from Eq. (36) that the parameters $t_{0}, t_{1}$, and $Y$ are the same parameters discussed in Sec. $I ; 4 t_{0}$ is the in-chain bandwidth, $4 t_{\perp}$ is the interchain bandwidth, and $4 t_{0} Y$ is the one-dimensional band gap. The actual band gap is an indirect gap of magnitude $4 t_{0} Y-4 t_{\perp}$. We note that the extreme simplicity of the band structure would be lost if further-neighbor interchain interactions were included. Then, the wave functions depend on $\overrightarrow{\mathrm{k}}_{\perp}$ as well as $k_{\|}$, and the two contributions to $E_{n}$ do not add simply.

\section{DIELECTRIC FUNCTION}

It is well known ${ }^{7,8}$ that, in a perfect crystal, the response of the crystal to a long-wavelength $(q \rightarrow 0)$ photon involves not only the macroscopic component of the charge density with small wave vector, $\vec{q}$, but also the mi- 
croscopic corrections of large-wave-vector, $\overrightarrow{\mathrm{q}}+\overrightarrow{\mathrm{G}}$, localfield fluctuations on the scale of the atoms. This results in a formula for the macroscopic dielectric function,

$$
\epsilon_{\mathrm{mac}}(\overrightarrow{\mathrm{q}}, \omega)=\left[\epsilon^{-1}(\overrightarrow{\mathrm{q}}, \overrightarrow{\mathrm{q}}, \omega)\right]^{-1} \text {. }
$$

The local-field corrections are associated with the inversion of the dielectric matrix $\epsilon\left(\overrightarrow{\mathrm{q}}+\overrightarrow{\mathrm{G}}, \overrightarrow{\mathrm{q}}+\overrightarrow{\mathrm{G}}^{\prime}, \omega\right)$ to obtain $\epsilon^{-1}\left(\overrightarrow{\mathrm{q}}+\overrightarrow{\mathrm{G}}, \overrightarrow{\mathrm{q}}+\overrightarrow{\mathrm{G}}^{\prime}, \omega\right)$.

Since $\epsilon\left(\overrightarrow{\mathrm{q}}+\overrightarrow{\mathrm{G}}, \overrightarrow{\mathrm{q}}+\overrightarrow{\mathrm{G}}^{\prime}\right)$ generally has important contri-

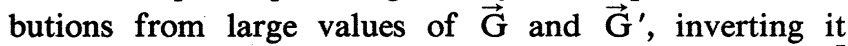
directly is not a promising approach. Hanke and Sham ${ }^{7}$ (HS) have presented a method to calculate $\epsilon(\omega)$ by using Wannier functions. In this paper we shall use their results; the reader is referred to Ref. 7 for details.

HS show that the inverse of the dielectric matrix is given by

$$
\begin{aligned}
\epsilon_{0} \epsilon^{-1}\left(\overrightarrow{\mathrm{q}}+\overrightarrow{\mathrm{G}}, \overrightarrow{\mathrm{q}}+\overrightarrow{\mathrm{G}}^{\prime}, \omega\right) \\
\quad=\delta_{\overrightarrow{\mathrm{G}} \overrightarrow{\mathrm{G}}},+v(\overrightarrow{\mathrm{q}}+\overrightarrow{\mathrm{G}}) \chi\left(\overrightarrow{\mathrm{q}}+\overrightarrow{\mathrm{G}}, \overrightarrow{\mathrm{q}}+\overrightarrow{\mathrm{G}}^{\prime}, \omega\right),
\end{aligned}
$$

where $v(\overrightarrow{\mathrm{q}})$ is the Coulomb potential which, at long wavelength, is certainly $v(\overrightarrow{\mathrm{q}})=4 \pi e^{2} / \Omega_{0} \epsilon_{0} q^{2} . \Omega_{0}$ is the volume of the unit cell. We have introduced a background dielectric constant $\epsilon_{0}$ to represent all other high-frequency response that is not included in the simplified Hamiltonian in Eq. (1). $\chi$ is the density correlation function, which is related to the irreducible correlation function $\widetilde{\chi}$ through the equation

$$
\begin{aligned}
\chi\left(\overrightarrow{\mathrm{q}}+\overrightarrow{\mathrm{G}}, \overrightarrow{\mathrm{q}}+\overrightarrow{\mathrm{G}}^{\prime \prime}, \omega\right)= & \tilde{\chi}\left(\overrightarrow{\mathrm{q}}+\overrightarrow{\mathrm{G}}, \overrightarrow{\mathrm{q}}+\overrightarrow{\mathrm{G}}^{\prime}, \omega\right) \\
+ & \sum_{\mathrm{G}^{\prime \prime}} \tilde{\chi}\left(\overrightarrow{\mathrm{q}}+\overrightarrow{\mathrm{G}}, \overrightarrow{\mathrm{q}}+\overrightarrow{\mathrm{G}}^{\prime \prime}, \omega\right) v\left(\overrightarrow{\mathrm{q}}+\overrightarrow{\mathrm{G}}^{\prime \prime}\right) \\
& \quad \times\left(\overrightarrow{\mathrm{q}}+\overrightarrow{\mathrm{G}^{\prime \prime}}, \overrightarrow{\mathrm{q}}+\overrightarrow{\mathrm{G}}^{\prime}, \omega\right) .
\end{aligned}
$$

For small wave vector $\overrightarrow{\mathrm{q}}$ and $\overrightarrow{\mathrm{G}}=\overrightarrow{\mathrm{G}}^{\prime}=0$, it is useful to consider another function $\hat{\chi}(\vec{q}, \omega)$, which satisfies the integral equation

$$
\begin{aligned}
\hat{\chi}(\overrightarrow{\mathrm{q}}, \omega)= & \tilde{\chi}(\overrightarrow{\mathrm{q}}, \omega) \\
& +\sum_{G \neq 0} \tilde{\chi}(\overrightarrow{\mathrm{q}}, \overrightarrow{\mathrm{q}}+\overrightarrow{\mathrm{G}}, \omega) v(\overrightarrow{\mathrm{q}}+\overrightarrow{\mathrm{G}}) \hat{\chi}(\overrightarrow{\mathrm{q}}+\overrightarrow{\mathrm{G}}, \overrightarrow{\mathrm{q}}, \omega),
\end{aligned}
$$

where $\hat{\chi}(\overrightarrow{\mathrm{q}}, \omega)$ is also related to

$$
\left[\chi\left(\overrightarrow{\mathrm{q}}+\overrightarrow{\mathrm{G}}, \overrightarrow{\mathrm{q}}+\overrightarrow{\mathrm{G}}^{\prime}, \omega\right)\right]_{\overrightarrow{\mathrm{G}}=\overrightarrow{\mathrm{G}}^{\prime}=0}=\chi(\overrightarrow{\mathrm{q}}, \omega)
$$

by the relation

$$
\chi(\overrightarrow{\mathrm{q}}, \omega)=\hat{\chi}(\overrightarrow{\mathrm{q}}, \omega) /[1-v(\overrightarrow{\mathrm{q}}) \hat{\chi}(\overrightarrow{\mathrm{q}}, \omega)] .
$$

Thus, we obtain

$$
\epsilon_{\mathrm{mac}}(\overrightarrow{\mathrm{q}}, \omega) \equiv \epsilon_{0}[1-v(\overrightarrow{\mathrm{q}}) \hat{\chi}(\overrightarrow{\mathrm{q}}, \omega)] .
$$

(Note, this quantity is only meaningful in the $|\vec{q}| \rightarrow 0$ limit.)

In terms of the Wannier functions of Eq. (3), $\hat{\chi}(\overrightarrow{\mathrm{q}}, \omega)$ is given by

$$
\hat{\chi}(\overrightarrow{\mathrm{q}}, \omega)=\sum_{s s^{\prime}} A_{s}(\overrightarrow{\mathrm{q}}) \hat{S}_{s s^{\prime}}^{-1}(\overrightarrow{\mathrm{q}}, \omega) A_{s^{\prime}}^{*}(\overrightarrow{\mathrm{q}}),
$$

where

$A_{s}(\overrightarrow{\mathrm{q}})=\int d^{3} r \phi_{v}^{*}(\overrightarrow{\mathrm{r}}-v \overrightarrow{\mathrm{a}}) e^{-i \overrightarrow{\mathrm{q}} \cdot \overrightarrow{\mathrm{r}}} \phi_{v^{\prime}}\left(\overrightarrow{\mathrm{r}}-\overrightarrow{\mathrm{R}}-v^{\prime} \overrightarrow{\mathrm{a}}\right)$,

and $s$ is the composite matrix index $s=\left(\vec{R}, v, v^{\prime}\right)$. The matrix $\hat{S}^{-1}$ is given by

$$
\begin{aligned}
\hat{S}^{-1}(\overrightarrow{\mathrm{q}}, \omega)=N^{(0)}(\overrightarrow{\mathrm{q}}, \omega)\{1- & {\left[\hat{V}(\overrightarrow{\mathrm{q}}, \omega)-\frac{1}{2} V^{x}(\overrightarrow{\mathrm{q}}, \omega)\right] } \\
& \left.\times N^{(0)}(\overrightarrow{\mathrm{q}}, \omega)\right\}^{-1},
\end{aligned}
$$

where $N^{(0)}$ is the polarization bubble of the form

$N_{\substack{\left.\overrightarrow{\mathrm{R}}_{1}, v_{1}, v_{1}^{\prime} \\ \overrightarrow{\mathrm{R}}_{2}, v_{2}, v_{2}^{\prime}\right\}}}^{(0)}(\overrightarrow{\mathrm{q}}, \omega)=\frac{2}{N} \sum_{n, n^{\prime}, \overrightarrow{\mathrm{k}}} U_{v_{1}}^{n^{*}(\overrightarrow{\mathrm{k}})} U_{v_{1}^{\prime}}^{n^{\prime}}(\overrightarrow{\mathrm{k}}+\overrightarrow{\mathrm{q}}) e^{i(\overrightarrow{\mathrm{k}}+\overrightarrow{\mathrm{q}})\left(\overrightarrow{\mathrm{R}}_{1}-\overrightarrow{\mathrm{R}}_{2}\right)} U_{v_{2}}^{n}(\overrightarrow{\mathrm{k}}) U_{v_{2}^{\prime}}^{n^{\prime *}}(\overrightarrow{\mathrm{k}}+\overrightarrow{\mathrm{q}}) \frac{f\left(n^{\prime}, \overrightarrow{\mathrm{k}}+\overrightarrow{\mathrm{q}}\right)-f(n, \overrightarrow{\mathrm{k}})}{E_{n^{\prime}}(\overrightarrow{\mathrm{k}}+\overrightarrow{\mathrm{q}})-E_{n}(k)-\hbar \omega-i \delta}$,

the Coulomb interaction

$$
\hat{V}_{s s^{\prime}}(\overrightarrow{\mathrm{q}})=\sum_{\overrightarrow{\mathrm{G}} \neq \overrightarrow{0}} A_{s}^{*}(\overrightarrow{\mathrm{q}}+\overrightarrow{\mathrm{G}}) v(\overrightarrow{\mathrm{q}}+\overrightarrow{\mathrm{G}}) A_{s^{\prime}}(\overrightarrow{\mathrm{q}}+\overrightarrow{\mathrm{G}}),
$$

and the exchange correction $V^{x}$ is given by

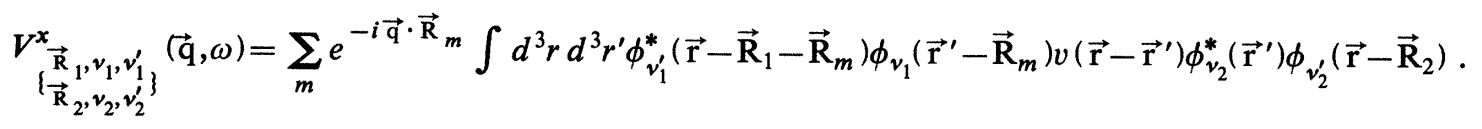

With this formal solution, the dielectric function can, in principle, be calculated from the wave functions and energies. Of these, the energies and the form of the wave functions for this model are derived in Sec. II.

\section{CALCULATED RESULTS}

To calculate the dielectric function, we also need to know the atomic wave function $\phi_{v}(\overrightarrow{\mathrm{r}}-v \overrightarrow{\mathrm{a}})$. Instead of us- ing an explicit form for $\phi_{v}$, we shall assume that $\phi_{v}$ does not overlap between neighboring sites, i.e.,

$$
A_{\left(\overrightarrow{\mathrm{R}} v, v^{\prime}\right)}(\overrightarrow{\mathrm{q}})=e^{-i \overrightarrow{\mathrm{q}} \cdot \overrightarrow{\mathrm{a}} v} \delta_{\overrightarrow{\mathrm{R}}, \overrightarrow{0}} \delta_{v^{\prime}}
$$

Later in this paper we shall relax this assumption and estimate the resulting correction. Using Eq. (16), we can calculate the matrix $\hat{S}^{-1}(\overrightarrow{\mathrm{q}}, \omega)$ of Eq. (12). 


\section{A. $\epsilon_{\mathrm{RPA}}^{(0)}(\overrightarrow{\mathrm{q}}, \omega)$}

If we neglect the local-field and exchange interaction, we have $\hat{S}^{-1}=N^{(0)}$. The dielectric function $\epsilon_{\mathrm{RPA}}^{(0)}$ is then given by

$\epsilon_{\mathrm{RPA}}^{(0)}(\overrightarrow{\mathrm{q}}, \omega)=\epsilon_{0}-\epsilon_{0} v(\overrightarrow{\mathrm{q}}) \sum_{s s^{\prime}} A_{s}(\overrightarrow{\mathrm{q}}) N^{(0)}(\overrightarrow{\mathrm{q}}, \omega) A_{s^{\prime}}^{*}(\overrightarrow{\mathrm{q}})$.

In the limit of small wave vector $\vec{q}$, we have

$$
\epsilon_{\mathrm{RPA}}^{(0)}(\overrightarrow{\mathrm{q}}, \omega)=\epsilon_{0}-\frac{2 \pi e^{2} q_{\|}^{2}}{\Omega_{0} q^{2} t_{0}} W_{1}(\omega),
$$

where

$$
\begin{aligned}
W_{1}(\omega)=\frac{y^{2}}{2 N} \sum_{\overrightarrow{\mathrm{k}}} \frac{2 t_{0}}{s_{\|}(k)} & {\left[\frac{1}{E_{1}(\overrightarrow{\mathrm{k}})-E_{0}(\overrightarrow{\mathrm{k}})-\omega-i \delta}\right.} \\
& \left.-\frac{1}{E_{0}(\overrightarrow{\mathrm{k}})-E_{1}(\overrightarrow{\mathrm{k}})-\omega-i \delta}\right] .
\end{aligned}
$$

We shall first examine this result at $\omega=0$. A little algebra leads to the result

$$
\begin{aligned}
\epsilon_{\mathrm{RPA}}^{(0)}(\overrightarrow{\mathrm{q}}, 0)=\epsilon_{0}+\epsilon_{1} & {\left[\frac{q_{\|}^{2}}{q^{2}}\right] F_{0}(y) } \\
& +X^{2}\left[\frac{z / 2-s_{\perp}\left(q_{\perp}\right)}{q}\right]^{2} F_{1}(y) \\
& \left.+O\left(q_{\|} a\right)^{4}+O\left(X_{\perp}^{4}\right)\right]
\end{aligned}
$$

where

$$
\epsilon_{1}=\frac{4 e^{2} a^{2}}{3 \Omega_{0} t_{0}}\left(\frac{1}{y}\right)^{2},
$$

$y=E_{g} / 4 t_{0}$, and $X_{\perp}=4 t_{\perp} / E_{g}=t_{\perp} / t_{0} y$. The structure $S_{\perp}$ is defined by $\mathrm{Eq}$. (3c). The functions $F_{i}(y)$ can be evaluated explicitly in terms of elliptic integrals and their derivatives. However, because the band gap $E_{\mathbf{g}}$ is much smaller than the in-chain bandwidth $4 t_{0}$ (i.e., $y=E_{g} / 4 t_{0} \cong 0.15$ $<1$ ), it is sufficient to evaluate them asymptotically as follows:

$$
\begin{aligned}
& F_{0}(y)=1+\frac{3}{4} y^{2}+O\left(y^{4}\right), \\
& F_{1}(y)=\frac{1}{5}-\frac{1}{8} y^{2}+O\left(y^{4}\right) .
\end{aligned}
$$

For $\hbar \omega>E_{g}, \epsilon_{\mathrm{RPA}}^{(0)}$ is complex. Thus, when $\bar{\omega}=\hbar \omega / 4 t_{0}$ is in the range $y<\bar{\omega}<1$, the imaginary part of $\epsilon_{\mathrm{RPA}}^{(0)}$ is

$$
\begin{aligned}
& \epsilon_{\mathrm{RPA}}^{(0) \prime \prime}(\overrightarrow{\mathrm{q}}, \omega) \\
& =\frac{3 \pi}{4} \epsilon_{1}\left[\frac{q_{\|}^{2}}{q^{2}} \frac{y^{4}}{\left[\left(1-\bar{\omega}^{2}\right)\left(\bar{\omega}^{2}-y^{2}\right)\right]^{1 / 2}}+O\left(X_{\perp}^{2}\right)\right] .
\end{aligned}
$$

All the results given above are based upon the zerooverlap approximation used in Eq. (16). To estimate the leading correction to these results, we next include the overlap between atomic wave functions on nearest- neighbor atoms. Thus, $A_{s}(\overrightarrow{\mathrm{q}})$ of Eq. (11) now has the value

$$
\begin{aligned}
A_{\left(\overrightarrow{\mathrm{R}}, v, v^{\prime}\right)}= & e^{-i \overrightarrow{\mathrm{q}} \cdot \overrightarrow{\mathrm{a}} v} \delta_{\overrightarrow{\mathrm{R}}, 0} \delta_{v^{\prime}}+B(\overrightarrow{\mathrm{q}}) \delta_{\overrightarrow{\mathrm{R}}, 0} \delta_{v 0} \delta_{v^{\prime} 1} \\
& +B^{*}(-\overrightarrow{\mathrm{q}}) e^{i \overrightarrow{\mathrm{q}} \cdot \overrightarrow{\mathrm{a}}} \delta_{\overrightarrow{\mathrm{R}},-\overrightarrow{1}} \delta_{v_{0}} \delta_{v^{\prime} 1},
\end{aligned}
$$

where $\overrightarrow{1}$ is the lattice vector between nearest-neighbor unit cells in the in-chain direction. Since we are interested in small $q a$, we only need to know the long-wavelength behavior of $B$. The orthonormality of the Wannier function insures that $B(0)=0$. We define the vector $\left.\overrightarrow{\mathrm{b}} \equiv i \nabla_{\overrightarrow{\mathrm{q}}} \boldsymbol{B}(\overrightarrow{\mathrm{q}})\right|_{\overrightarrow{\mathrm{q}}=0}$ which determines the leading $\overrightarrow{\mathrm{q}}$ dependence of the overlap. At zero frequency, including $B \neq 0$ gives the following correction to $\epsilon_{\mathrm{RPA}}^{(0)}(\overrightarrow{\mathrm{q}}, 0)$ in Eq. (19):

$$
\Delta \epsilon_{\mathrm{RPA}}^{(0)}=\epsilon_{1}\left(\frac{\overrightarrow{\mathrm{b}} \cdot \overrightarrow{\mathrm{q}}}{q a}\right)^{2} \frac{3}{2} y^{2} \ln (4 / y) .
$$

For $y<0.15$, this is less than a $10 \%$ correction. At nonzero frequencies, the correction to the imaginary part of the dielectric function is

$$
\begin{aligned}
\Delta \epsilon_{\mathrm{RPA}}^{(0)}= & \frac{3 \pi}{4} \epsilon_{1}\left[\frac{\overrightarrow{\mathrm{b}} \cdot \overrightarrow{\mathrm{q}}}{q a}\right]^{2} \cdot\left(\frac{1}{\bar{\omega}}\right) \frac{y^{4}}{\left(1-y^{2}\right)^{2}} \\
& \times\left(1-\bar{\omega}^{2}\right)^{1 / 2}\left(\omega^{2}-y^{2}\right)^{1 / 2} .
\end{aligned}
$$

This correction is negligible for frequencies near the band gap, but becomes appreciable for higher frequencies. The same is true of corrections to the real part of $\epsilon_{\mathrm{RPA}}^{(0)} \epsilon_{\mathrm{RPA}}^{(0)}$.

Since we have until now neglected the local-field effect, we have obtained a square-root singularity in $\epsilon^{\prime \prime}$ for frequencies near the band gap, $\epsilon_{\mathrm{RPA}}^{(0)} \sim\left(\bar{\omega}^{2}-y^{2}\right)^{-1 / 2}$. Similar behavior is also observed in the optical-absorption coefficient.

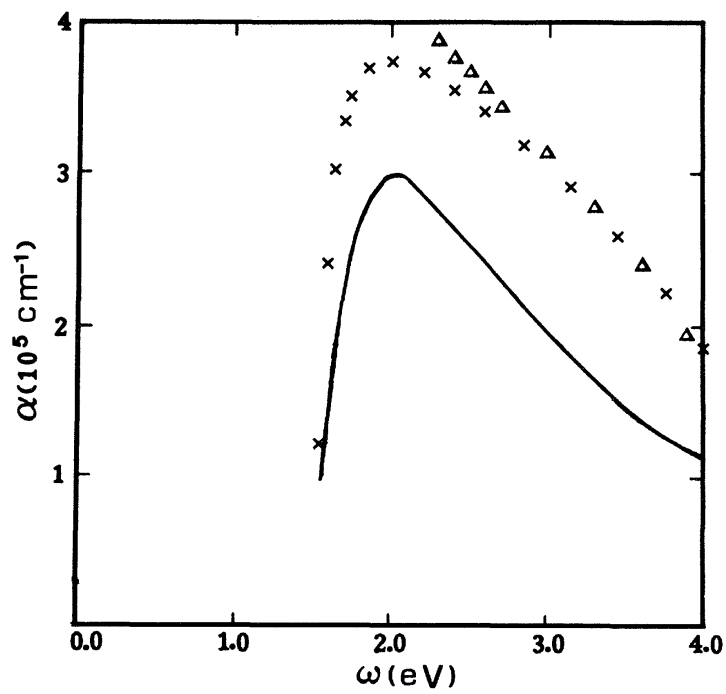

FIG. 2. Optical absorption in trans- $(\mathrm{CH})_{x}$. Solid line is the experimental result from Ref. 11, the crosses and triangles are the theoretical results with and without local-field corrections. See the text for details. 


$$
\alpha^{(0)}(\omega)=\frac{\omega}{c \sqrt{2}}\left\{\left[\left|\epsilon_{\mathrm{RPA}}^{(0)}(\omega)\right|-\epsilon_{\mathrm{RPA}}^{(0) \prime}(\omega)\right]^{1 / 2}\right.
$$

The numerical results are shown in Fig. 2 as the triangles for $\epsilon_{0}=3, y=0.15,4 t_{0}=10 \mathrm{eV}, a=1.23 \AA$, and $a^{3} / \Omega_{0}$ $=0.048$.

The local field $\hat{V}$ and/or the exchange interaction $V^{x}$ in Eq. (12), produce a qualitative change in the behavior for frequency near the band gap.

\section{B. $\epsilon_{\mathrm{RPA}}(\overrightarrow{\mathrm{q}}, \omega)$ with local field}

To simplify the analysis in this section, we will once again ignore small effects of the overlap between nearestneighbor wave functions [as in Eq. (16)] and of interchain coupling. As before, this approximation is valid as long as the frequency is not much greater than $E_{g}$. This approximation allows us to do most of the calculations analytically. First we treat the local-field corrections ignoring the exchange interactions. We will consider the exchange interactions later.

Because we are only interested in small wave vectors, the property of Eq. (16), and the condition

$$
\sum_{v} U_{v}^{n *}(\overrightarrow{\mathrm{k}}) U_{v}^{n^{\prime}}(\overrightarrow{\mathrm{k}})=\left(1-\delta_{n n^{\prime}}\right),
$$

the matrix $\hat{S}^{-1}$ in Eq. (12) is of the relatively simple form,

$$
\begin{aligned}
\hat{S}^{-1}(\overrightarrow{\mathrm{q}}, \omega)=N^{(0)}(\overrightarrow{\mathrm{q}}, \omega)+ & N^{(0)}(\overrightarrow{\mathrm{q}}, \omega)\left[1-\hat{V}(0) N^{(0)}(0, \omega)\right]^{-1} \\
& \times \hat{V}(0) N^{(0)}(\overrightarrow{\mathrm{q}}, \omega),
\end{aligned}
$$

and Eq. (14) becomes

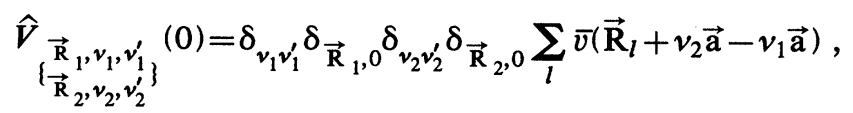

where

$$
\begin{aligned}
\bar{v}\left(\overrightarrow{\mathrm{R}}_{l}+v_{2} \overrightarrow{\mathrm{a}}-v_{1} \overrightarrow{\mathrm{a}}\right)=\int & d^{3} r d^{3} r^{\prime}\left|\phi_{v_{1}}(\overrightarrow{\mathrm{r}})\right|^{2} \\
& \times\left|\phi_{v_{2}}\left(\overrightarrow{\mathrm{r}}^{\prime}-\overrightarrow{\mathbf{R}}_{l}\right)\right|^{2} v\left(\overrightarrow{\mathbf{r}}-\overrightarrow{\mathbf{r}}^{\prime}\right) .
\end{aligned}
$$

More explicitly, we have

$$
\epsilon_{\mathrm{RPA}}(\overrightarrow{\mathrm{q}}, \omega)=\epsilon_{0}-\frac{2 \pi e^{2} q_{\|}^{2}}{\Omega_{0} q^{2} t_{0}}\left[W_{1}(\omega)+\frac{V_{c} W_{2}^{2}(\omega)}{1-V_{c} W_{3}(\omega)}\right],
$$

where $W_{1}(\omega)$ is given by Eq. (18) as

$$
\begin{aligned}
W_{2}(\omega)=\frac{y}{N} \sum_{k} \frac{2 t_{0}}{s_{\|}^{2}(\overrightarrow{\mathrm{k}})} & \frac{1}{E_{1}(\overrightarrow{\mathrm{k}})-E_{0}(\overrightarrow{\mathrm{k}})-\omega-i \delta} \\
& \left.-\frac{1}{E_{0}(k)-E_{1}(\overrightarrow{\mathrm{k}})-\omega-i \delta}\right),
\end{aligned}
$$

$$
\begin{aligned}
W_{3}(\omega)=\frac{4 t_{0}}{N} \sum_{k} & \frac{1}{E_{1}(k)-E_{0}(\overrightarrow{\mathrm{k}})-\omega-i \delta} \\
& \left.-\frac{1}{E_{0}(\overrightarrow{\mathrm{k}})-E_{1}(\overrightarrow{\mathrm{k}})-\omega-i \delta}\right],
\end{aligned}
$$

and

$$
V_{c}=\frac{1}{4 t_{0}} \sum_{l}\left[\bar{v}\left(\overrightarrow{\mathbf{R}}_{l}\right)-\bar{v}\left(\overrightarrow{\mathbf{R}}_{l}+\overrightarrow{\mathbf{a}}\right)\right] .
$$

Thus, we are able to combine all of the local-field effects into one dimensionless parameter $V_{c}$. There are simple relations between the real and imaginary parts of $W_{i}(\omega)$. They are given in the Appendix. The imaginary part of $\epsilon_{\mathrm{RPA}}(\overrightarrow{\mathrm{q}}, \omega)$ is given by

$$
\begin{aligned}
\epsilon_{\mathrm{RPA}}^{\prime \prime}(\overrightarrow{\mathrm{q}}, \omega)= & \frac{3 \pi}{16} \epsilon_{1} \frac{q_{\|}^{2}}{q^{2}} \frac{y^{4}}{\bar{\omega}^{5}}\left[\left(\omega^{2}-y^{2}\right)\left(1-\bar{\omega}^{2}\right)\right]^{1 / 2} \\
& \times \frac{\left[1+\left(4 V_{c} / \pi\right) \ln (4 / y)\right]^{2}}{V_{c}^{2}+P^{2}}
\end{aligned}
$$

where

$$
P=\frac{1}{W_{3}^{\prime \prime}}-V_{c} \frac{W_{3}^{\prime}}{W_{3}^{\prime \prime}} .
$$

Thus, instead of having a singularity when frequency is near the band gap [as does $\epsilon_{\mathrm{RPA}}^{(0)}(\omega)$ ], we find that $\epsilon_{\mathrm{RPA}}^{\prime \prime}$ approaches 0 near the band gap, thus a finite peak in the optical-absorption results. This peak is fairly asymmetrical. We have numerically calculated the opticalabsorption coefficient $\alpha(\omega)$ of Eq. (25) by using $\epsilon_{\mathrm{RPA}}(\omega)$. The result is plotted in Fig. 2 (the $X$ 's). The parameters are the same as before with the addition of $V_{c}=0.25$.

We would like to emphasize that the numerical result is very insensitive to the value of $V_{c}$ within the range $0.1<V_{c}<2$. Only a very slight shift of the peak position occurs as a function of $V_{c}$.

The static value of $\epsilon_{\mathrm{RPA}}$ is given by the expression

$$
\begin{aligned}
& \epsilon_{\mathrm{RPA}}(\omega=0) \\
& =\epsilon_{0}+\epsilon_{1}\left(\frac{q_{\|}}{q}\right)^{2}\left(1+\frac{3}{4} y^{2}-\frac{6 V_{c}}{\pi+4 V_{c} \ln (4 / y)}\right) .
\end{aligned}
$$

We shall now estimate the effects of exchange interaction $V^{x}$ in Eq. (12).

\section{Exchange correction}

By making the same approximation leading to Eqs. (16) and (29), we find out $V^{x}$ of Eq. (15) to be

$$
\begin{aligned}
& V_{\substack{V_{\mathbf{R}_{2}}, \vec{R}_{1}, v_{1}, v_{1}^{\prime} \\
\vec{R}_{2_{2}}, v_{2}^{\prime}}}(q=0, \omega) \\
& =\delta_{v_{1} v_{2}{ }_{v_{1}^{\prime} v_{2}^{\prime}}} \delta_{\overrightarrow{\mathrm{R}}_{1}, \overrightarrow{\mathrm{R}}} \bar{v}\left(\overrightarrow{\mathrm{R}}_{1}+v_{1}^{\prime} \overrightarrow{\mathrm{a}}-v_{1} \overrightarrow{\mathrm{a}}\right) .
\end{aligned}
$$

This has many nonzero matrix elements, and hence makes 
the matrix inversion rather complicated. However, we argue that once the $1 /\left(\hbar \omega-E_{g}\right)^{1 / 2}$ singularity has been removed via local-field effects, the effect of the exchange interaction is rather small. To see this, we imagine that the wave function $\phi_{v}$ is very localized, and therefore $\bar{v}(0) \gg \bar{v}(\overrightarrow{\mathbf{R}}+v \overrightarrow{\mathbf{a}})$ for $\overrightarrow{\mathbf{R}}+v \overrightarrow{\mathbf{a}} \neq 0$. [Actually, we expect $\bar{V}(v \overrightarrow{\mathrm{a}})$ to be approximately one-half to one-fourth of $\bar{V}(0)$.] In this limit, $V^{x}(q=0) \approx \frac{1}{2} \hat{V}(q=0)$; the effect of $V^{x}$ is thus to reduce the value of $V_{c}$ to a smaller effective value, $V_{c}^{\text {eff }}=V_{c}-V_{x}$. Since $\epsilon_{\mathrm{RPA}}$ is not very sensitive to the value of $V_{c}$, this does not affect our results in any important way. Taking into account the full matrix structure of $V_{x}$ will, of course, have more effect than this; it will result in some slight shifts in the shape of the absorption spectrum. However, as best we can determine, none of these effects are very large.

\section{IMPLICATION FOR $(\mathrm{CH})_{x}$}

The band gap in trans $-(\mathrm{CH})_{x}$ is about $1.6-1.9 \mathrm{eV}, 1^{10}$ while the bandwidth is on the order of $10 \mathrm{eV}$, and, therefore $y \cong 0.16$. Thus, we are justified in keeping only lowest-order terms in the expansion of $\epsilon_{\mathrm{mac}}$ in powers of $y$. Moreover, since $x \lesssim 0.1$, we can also ignore the contribution from the interchain coupling.

The characteristic values for trans $-(\mathrm{CH})_{x}$ are $a=1.23$ $\AA$ and $\Omega_{0}=38.4 \AA^{3}$. If we take $\epsilon_{0}=3$, and $\bar{v}(0) \cong e^{2} / a \epsilon_{0}$ $\cong 4 \mathrm{eV}$, then $V_{c} \cong 0.25$. The static RPA dielectric constant with or without taking into account local-field effects gives us $\epsilon_{\mathrm{RPA}}(\omega=0)=13.5$ and $\epsilon_{\mathrm{RPA}}^{0}(\omega=0)=16.5$. This result agrees well with the experiment ${ }^{11}$ in pristine $(\mathrm{CH})_{x}$.

The experimental results for the optical-absorption coefficient $\alpha(\omega)$ are shown in Fig. 2 as the solid line. The results calculated with and without local-field effects are represented by the crosses and triangles, respectively. The magnitude of calculated results is in fairly good agreement with experimental data. However, this agreement should not be taken too seriously, as it is sensitive to the precise value of $\epsilon_{0}$ and should contain correction due to the inhomogeneity of the material on micrometer length scales. There are certain discrepancies at high frequency. This is to be expected as the neglect of interchain coupling and of the overlap between Wannier functions on neighbor sites in the integral $A_{s}(\overrightarrow{\mathrm{q}})$ of Eq. (11) is justified only for frequency near the gap. Appreciable corrections are expected at high frequency. We have also calculated $\epsilon_{\mathrm{RPA}}(\omega)$ and $\epsilon_{\mathrm{RPA}}^{(0)}(\omega)$ for cis- $(\mathrm{CH})_{x}$ as shown in Fig. 3. We take as representative parameters $y=0.19, \epsilon_{0}=3$, $4 t_{0}=10 \mathrm{eV}, a=1.1 \AA$, and $a^{3} / \Omega_{0}=0.036$. The experimental result shown is obtained from Ref. 11. The multiple peaks observed near $2.1 \mathrm{eV}$ are presumably due to multiple-phonon emission.

We would like to emphasize that in our theory, $V_{c}$ is the only parameter whose value is not at least reasonably well known. There are no other adjustable parameters. Moreover, the value of $\alpha(\omega)$ is quite insensitive to the value of $V_{c}$, especially for frequency near the band gap. This leads us to conclude that a more accurate calculation which takes into account the exchange interaction and uses more realistic Wannier functions would only differ

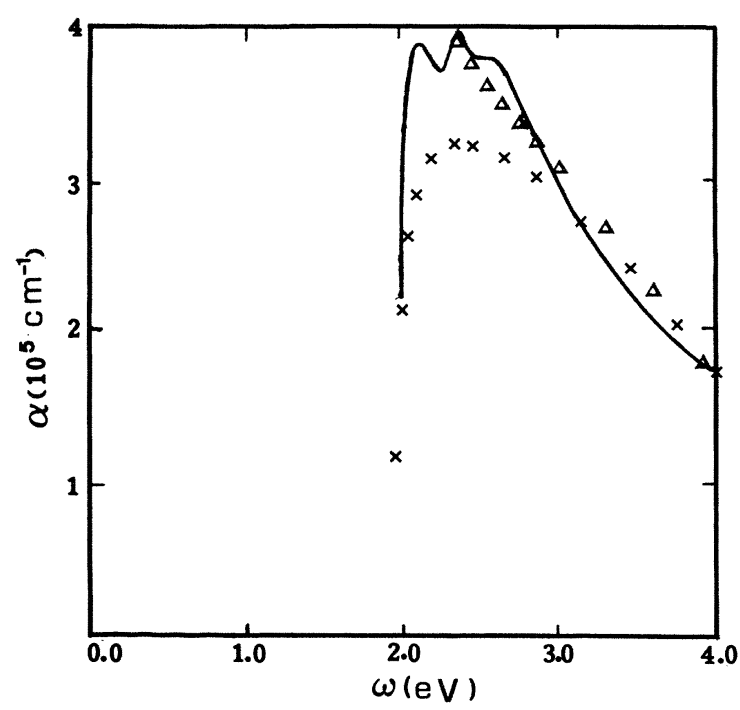

FIG. 3. Optical absorption in cis- $(\mathrm{CH})_{x}$. See Fig. 2 and the text.

substantially from the present calculation of $\alpha(\omega)$ at high frequencies.

The other application of our results to $(\mathrm{CH})_{x}$ that is worth mentioning is the effect of screening on the binding energy of a charged soliton to an oppositely charged impurity. The charged soliton ${ }^{4}$ is the lowest-energy charged excitation in $(\mathrm{CH})_{x}$. In the most commonly studied case, solitons are introduced by interstitial doping. Thus, the interaction between the dopant and the impurity is of central importance. For our purposes, the details of the soliton structure is unimportant. All that we need to know is that a soliton is confined to a single chain and its charge density is spread out over a distance, $2 \xi_{0}=2 a / y$, which is large compared to $a$. Because of this, the dopant ion can be treated as a point charge. Naively, one might also expect that the large extent of the soliton would imply that the soliton-dopant interaction is screened by the full macroscopic dielectric function. In fact, this is not the case since, as implied by Eq. (1), the full dielectric screening occurs only on distances that are large compared to $\xi_{0}$. Nonetheless, an approximate estimate (most probably a lower bound) of the binding of a soliton to an impurity can be made by ignoring this complication. To further simplify matters, we will approximate the soliton chargedensity form factor by a square distribution,

$$
\rho(x) \approx e \Theta\left(\xi_{0}^{2}-x^{2}\right) / 2 \xi_{0},
$$

although the actual form factor is somewhat more complicated. $^{12}$ With these approximations it is easy to see that the binding energy of a soliton to an impurity is

$$
\begin{aligned}
E_{b} & =\left(\frac{e^{2}}{\epsilon_{0} \xi_{0}}\right] \sinh ^{-1}\left(\xi_{0} / d^{\mathrm{eff}}\right) \\
& \approx\left(\frac{e^{2}}{\epsilon_{0} \xi_{0}}\right] \ln \left(2 \xi_{0} / d^{\mathrm{eff}}\right),
\end{aligned}
$$

where $d$ is the distance between the impurity and the chain, $d^{\text {eff }}=d\left[1+\left(\epsilon_{1} / \epsilon_{0}\right)\right]^{1 / 2}$, and the second expression is valid for $\xi_{0} \gg d^{\text {eff }}$. Notice the weak (logarithmic) 
dependence on $d$. For $d=2.2 \AA$, we find that $E_{b} \approx 0.5$ eV. Recent experiments ${ }^{13}$ on photoinduced optical absorption in $(\mathrm{CH})_{x}$ can be interpreted as evidence of a soliton binding energy of $\sim 0.4 \mathrm{eV}$, in good agreement with our theoretical estimate. Of course, with such a large binding energy, it is not justified to treat the interaction potential in first-order perturbation theory (which is effectively what we have done when we assigned a rigid charge density to the soliton). Thus, one should not overinterpret the agreement between theory and experiment.

\section{CONCLUSIONS}

We have calculated the optical dielectric function for polyacetylene in the RPA, including local-field corrections. We believe the results are semiquantitatively correct at long wavelengths and for frequencies not much in excess of the gap. This conclusion is supported by the comparison between our results and experiment for the optical-absorption coefficient.

Note that in all of our calculations the lattice has been treated as rigid. Of course, for energies below the band gap, lattice-dynamical effects are necessarily important. However, perhaps surprisingly, our calculations show that at least the general shape of the optical absorption can be understood without taking lattice dynamics into account at all.

\section{ACKNOWLEDGMENTS}

We thank Dr. A. Muramatsu for useful discussions of the Sham and Hanke formalism and Dr. A. Epstein for important help in interpreting experiments. This work was partially supported by a Coal and Energy Research grant from the Virginia Polytechnic Institute Research Division.
APPENDIX: $W_{i}(\omega)$

If we neglect the interchain coupling, the imaginary parts of $W_{i}(\omega)$ of Eqs. (18) and (28) can easily be calculated, they are given by

$$
\begin{aligned}
& W_{3}^{\prime \prime}(\omega)=\operatorname{Im} W_{3}(\omega)=-2 \bar{\omega}\left[\left(\bar{\omega}^{2}-y^{2}\right)\left(1-\bar{\omega}^{2}\right)\right]^{-1 / 2}, \\
& W_{2}^{\prime \prime}(\omega)=-\frac{y}{\bar{\omega}}\left[\left(\bar{\omega}^{2}-y^{2}\right)\left(1-\bar{\omega}^{2}\right)\right]^{-1 / 2},
\end{aligned}
$$

and

$$
W_{1}^{\prime \prime}(\omega)=W_{2}^{\prime \prime 2} / W_{3}^{\prime \prime} \text {. }
$$

The real parts of $W_{i}(\omega)$, namely $W_{1}^{\prime}(\omega)$, are related to each other through the following relationships:

$$
\frac{W_{1}^{\prime}}{W_{1}^{\prime \prime}}-\frac{W_{2}^{\prime}}{W_{2}^{\prime \prime}}=\frac{4 \bar{\omega}^{-2}}{\pi W_{3}^{\prime \prime}} \int_{0} \frac{d k}{s_{\|}^{3}(k)}
$$

and

$$
\frac{W_{2}^{\prime}}{W_{2}^{\prime \prime}}-\frac{W_{3}^{\prime}}{W_{3}^{\prime \prime}}=\frac{4}{\pi} \frac{1}{W_{3}^{\prime \prime}} \int_{0} \frac{d k}{s_{\|}(k)},
$$

where $s_{\|}(k)$ is defined in Eq. (3). Because $y=E_{g} / 4 t_{0}$ $\ll 1$, Eqs. (A4) and (A5) can be evaluated approximately, and are given by

$$
\frac{W_{1}^{\prime}}{W_{2}^{\prime \prime}}-\frac{W_{2}^{\prime}}{W_{2}^{\prime \prime}}=\frac{4 \bar{\omega}^{2}}{\pi W_{3}^{\prime \prime}}\left(\frac{1}{2} \ln (4 / y)+\frac{1}{y^{2}}\right)
$$

and

$$
\frac{W_{2}^{\prime}}{W_{2}^{\prime \prime}}-\frac{W_{3}^{\prime}}{W_{3}^{\prime \prime}}=\frac{4}{\pi} \frac{1}{W_{3}^{\prime \prime}} \ln (4 / y) .
$$

${ }^{1}$ W. P. Su, J. R. Schrieffer, and A. J. Heeger, Phys. Rev. B 22 , 2099 (1980).

${ }^{2}$ N. Suzuki, M. Ozuki, S. Etemad, A. J. Heeger, and A. G. MacDiarmid, Phys. Rev. Lett. 45, 1209 (1980).

${ }^{3}$ Z. Vardeny, J. Ornstein, and G. L. Baker (unpublished); Z. Vardeny (private communication).

${ }^{4}$ S. Kivelson, T. K. Lee, Y. R. Lin Liu, J. Peschel, and Lu Yu, Phys. Rev. B 25, 4173 (1983).

5J. T. Gammel and J. A. Krumhansl, Phys. Rev. B $\underline{24,} 1035$ (1981).

${ }^{6}$ K. Maki and M. Nakahara, Phys. Rev. B $\underline{23}, 5005$ (1981).

${ }^{7}$ W. Hanke and L. S. Sham, Phys. Rev. B $\underline{12}, 4501$ (1975).

${ }^{8}$ W. Hanke, Adv. Phys. 27, 287 (1978).
${ }^{9}$ C. R. Fincher, Jr., C.-E. Chen, A. J. Heeger, A. G. MacDiarmid, and J. B. Hastings, Phys. Rev. Lett. 48, 100 (1982).

${ }^{10}$ A. Feldblum, J. H. Kaufman, S. Etemad, A. J. Heeger, T.-C. Chung, and A. G. MacDiarmid, Phys. Rev. B 26, 815 (1982).

${ }^{11}$ C. R. Fincher, Jr., M. Ozaki, M. Tanaka, D. Peebles, L. Lauchlan, A. J. Heeger, and A. G. MacDiarmid, Phys. Rev. B 20, 1589 (1979).

${ }^{12}$ The actual charge density is more nearly approximated by the following expression:

$$
\rho(x)=e \xi_{0}^{-1} \cos (\pi x / 2 a) \operatorname{sech}^{2}\left(x / \xi_{0}\right) .
$$

13J. Ornstein and G. L. Baker, Phys. Rev. Lett. 49, 1043 (1982). 